Editorial

\title{
Tuberculosis care in 2013 and beyond: 20 years after the birth of the DOTS strategy
}

\author{
Akihiro Seita
}

TIME Magazine recently featured tuberculosis in its cover story, "Contagion: why drug-resistant tuberculosis threatens us all" [1]. It has been almost 20 years since such an international news magazine featured tuberculosis as cover story. The New England Journal of Medicine also featured a review article on tuberculosis recently [2]. It seems that the global interest in tuberculosis is reviving. This is exciting news for those working for tuberculosis care. Tuberculosis is still prevalent in the world, and drug-resistant tuberculosis is on the rise. Thus what should we do better and more to benefit from this increasing attention to tuberculosis? It becomes more important to reflect on this when we commemorate World Tuberculosis Day on 24 March. One answer lies in how we now understand the DOTS strategy.

DOTS has been the main thrust of modern tuberculosis care. The strategy was developed almost 20 years ago and constituted the foundations for the Stop TB Strategy in 2006. The Stop TB Strategy comprises six components providing a multi-pronged approach to address the key challenges of tuberculosis, and DOTS has remained at the core of the Stop TB Strategy. DOTS is a well-structured package for diagnosis and treatment of tuberculosis patients, including management and supportive systems. The impact of DOTS is evident: "an estimated 20 million people are alive today as a direct result of structured tuberculosis care" [3].
The philosophy ofDOTS - namely the logical sequence behind the development of the strategy, and not only the technical framework - is also universal. In support this, we recently published two articles on the use of quarterly cohort analysis, which is the core monitoring tool for tuberculosis care, to improve diabetes and hypertension care $[4,5]$. The articles received positive responses from the global health community. The articles describe the translation of the lessons learned in tuberculosis care into care for noncommunicable diseases, a rapidly growing worldwide epidemic. Although our experience is relatively limited, our stance on the universality of the philosophy of the DOTS strategy is solid. With this in mind, we wrote an editorial, "All you need to know in public health we can learn from tuberculosis care: lessons for noncommunicable disease" [6].

Still, it is very important to realize that the DOTS strategy is now 20 years old. No such package of products and services can maintain its effectiveness over the decades. The tuberculosis situation and tuberculosis care 20 years ago is quite different from now. Let us think of the case detection rate (CDR), which is one of the key indicators for tuberculosis care as an example. In the WHO Eastern Mediterranean Region (EMR), there were around 1 million prevalent cases of tuberculosis in 2011, however, CDR has remained around $62 \%$ in the past fewyears [3]. Of course, the reasons for this are numerous. Moreover, we all know that people working in tuberculosis care make tremendous efforts to improve the situation. Nevertheless, the question is, "Can the DOTS strategy continue to improve CDR to say $70 \%, 80 \%$ or $90 \%$, as it helped do in bringing CDR up to $62 \%$ in EMR?" This needs careful and strategic thinking, but the answer is probably no. The effectiveness of DOTS relies mostly on two assumptions: tuberculosis patients are homogeneously present throughout the communities, and thus, patients are accessible through passive case detection. These two assumptions are relevant in relation those who have already been diagnosed, however they may not be relevant in the case of the remaining $37 \%$ of undiagnosed patients. Blindly following DOTS may not now result in higher case detection, and thus we may need to be free of the constraints of a dogmatic approach.

The world (except the World Health Organization African Region) is on track to meet the tuberculosisrelated Millennium Development Goals (MDGs). The global tuberculosis community is now working for the post-2015 development agenda that will succeed the MDGs. The post2015 tuberculosis strategy will also be formulated accordingly. We need to help global efforts by noting the value of DOTS but more importantly recognizing the limitations of this 20-year-old strategy. EMR will also benefit from this global rethinking. Only then can the Region address the challenges of improving its case detection and tackling drug-resistant tuberculosis. 


\section{References}

1. Krista Mahr. Contagion: Why drug-resistant tuberculosis threatens us all. TIME, 4 March, 2013.

2. Zumla A et al. Tuberculosis, current concept. New England Journal of Medicine, 2013, 368:745-755.

3. Global tuberculosis report 2012. Geneva, World Health Organization, 2012 (WHO/HTM/TB/2012.6).

4. Khader A et al. Cohort monitoring of persons with hypertension: an illustrated example from a primary health care clinic for Palestine refugees in Jordan. Tropical Medicine \& International Health, 2012, 17:1163-1170.
5. Khader A et al. Cohort monitoring of persons with diabetes mellitus in a primary health care clinic for Palestine refugees in Jordan. Tropical Medicine \& International Health, 2012, 17:1569-1576.

6. Seita A, Harries AD. All you need to know in public health we can learn from tuberculosis care: lesions for non-communicable disease. International Journal of Tuberculosis and Lung Disease, 2013, 17:429-430. 